\title{
Accretion Flow Dynamics During 1999 Outburst of XTE J1859+226 - Modeling of Broadband Spectra and Constraining the Source Mass
}

\author{
A. Nandi ${ }^{1}$ • S. Mandal ${ }^{2}$ • H. Sreehari ${ }^{1,3}$ • \\ Radhika D. ${ }^{4}$ - S. Das ${ }^{5}$ - I. Chattopadhyay ${ }^{6}$ •

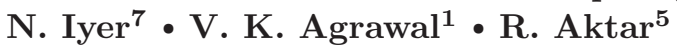

\begin{abstract}
We examine the dynamical behavior of accretion flow around XTE J1859+226 during the 1999 outburst by analyzing the entire outburst data $(\sim 166$ days $)$ from RXTE Satellite. Towards this, we study the hysteresis behavior in the hardness intensity diagram (HID) based on the broadband $(3-150 \mathrm{keV})$ spectral modeling, spectral signature of jet ejection and the evolution of Quasiperiodic Oscillation (QPO) frequencies using the twocomponent advective flow model around a black hole. We compute the flow parameters, namely Keplerian accretion rate $\left(\dot{m}_{d}\right)$, sub-Keplerian accretion rate $\left(\dot{m}_{h}\right)$, shock location $\left(r_{s}\right)$ and black hole mass $\left(M_{b h}\right)$ from the spectral modeling and study their evolution along the q-diagram. Subsequently, the kinetic jet power is computed as $L_{\text {jet }}^{\text {obs }} \sim 3-6 \times 10^{37} \mathrm{erg} \mathrm{s}^{-1}$ during one of the
\end{abstract}

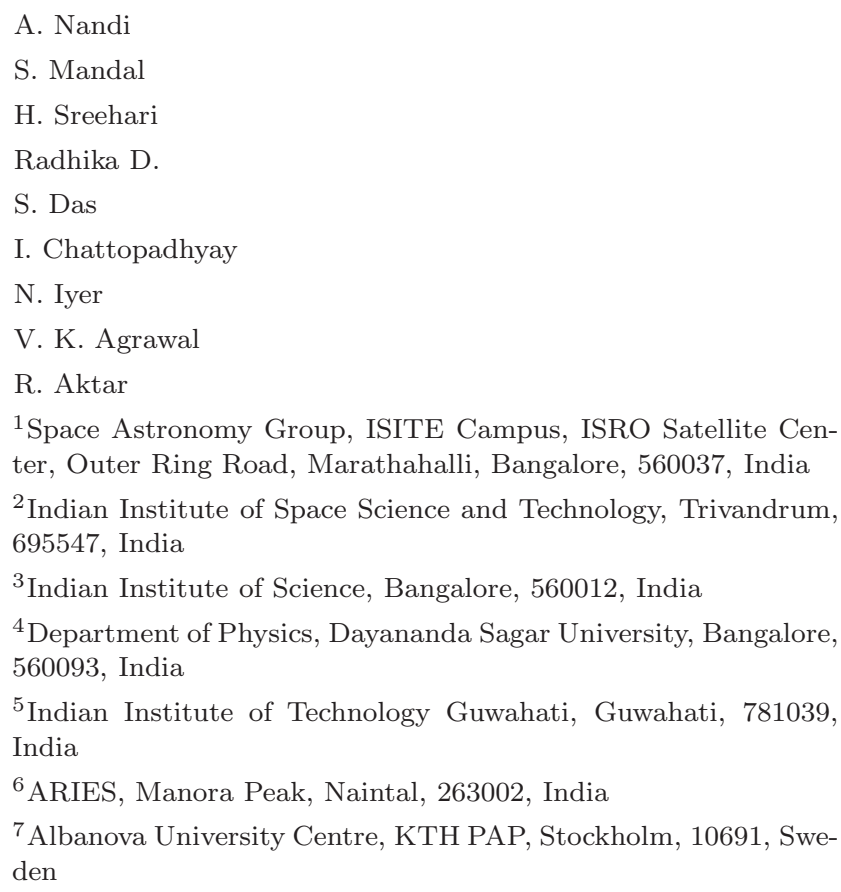

observed radio flares which indicates that jet power corresponds to $8-16 \%$ mass outflow rate from the disc. This estimate of mass outflow rate is in close agreement with the change in total accretion rate $(\sim 14 \%)$ required for spectral modeling before and during the flare. Finally, we provide a mass estimate of the source XTE J1859+226 based on the spectral modeling that lies in the range of $5.2-7.9 M_{\odot}$ with $90 \%$ confidence.

Keywords accretion, accretion discs - black hole physics - radiation mechanisms: non-thermal - X-rays: binaries - ISM : jets and outflows - stars : black holes

\section{Introduction}

In the quest of the accretion-ejection phenomena in the realm of strong gravity, astrophysical black holes systems are considered to be the ideal laboratories. Moreover, most of the galactic black hole sources (GBHs) exhibit rich 'spectro-temporal' variabilities in $\mathrm{X}$-rays that carry the signature of accretion-ejection activity (Mirabel \& Rodríguez 1994; Feroci et al. 1999;

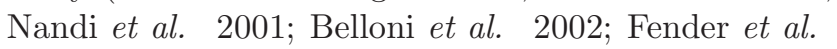
2009; Aktar et al. 2015, 2017; Sreehari et al. 2018). Extensive X-ray observations by Rossi-X-ray Timing Explorer (RXTE) revealed a direct correlation between the evolution of Quasi-Periodic Oscillation (QPO) frequencies and the spectral energy distribution in black hole X-ray binaries (XRBs). In case of outbursting sources, it is observed that the QPO frequency increases during the rising phase as the source transits from hard to intermediate states and subsequently QPOs disappear during soft states (Belloni et al. 2002, 2005; Chakrabarti et al. 2008; Nandi et al. 2012; Radhika \& Nandi 2014; Iver et al. 2015a, and references therein). On the other hand, a reverse trend of QPO frequency variation is observed during the 
declining phase of the outburst before the source transits to its quiescent phase (Belloni et al. 2005; Nandi et al. 2012; Debnath et al. 2013, references therein). In general, the hardness intensity diagram (HID) of GBH sources during the outburst exhibits a unique feature in the form of a 'q-shape' hysteresis loop known as 'q-diagram' (Maccarone \& Coppi 2003; Homan \& Belloni 2005; Remillard \& McClintock 2006; Nandi et al. 2012; Radhika \& Nandi 2014). In general, GBHs also display weak radio activities (Fender 2001; Corbel \& Fender 2002) in low-hard states (LHS) and hard-intermediate states (HIMS) during the rising phase of the outburst. In addition, a correlation is also observed between the radio and X-ray luminosities for several black hole sources Corbel et al. 2003; Fender \& Gallo 2014). Relativistic ejections in the form of radio flares are observed when the source transits from its hard-intermediate state (HIMS) to the soft-intermediate state (SIMS) Brocksopp et al. 2002; Corbel et al. 2003, 2004; Fender et al. 2004; Homan \& Belloni 2005; Fender et al. 2009; Miller-Jones 2012). These relativistic ejections (i.e., jets) are associated either with type A/B QPOs (Fender et al. 2004, 2009; Soleri et al. 2008) or no QPO with a signature of soft spectrum (Vadawale et al. 2001; Radhika \& Nandi 2014; Radhika et al. 2016). Interestingly, type C QPOs are generally observed in both LHS and HIMS (Casella et al. 2004; Homan \& Belloni 2005) during the outbursting phase of the black hole sources.

Numerous theoretical and phenomenological attempts were made to understand the physical mechanisms responsible for the above mentioned X-ray characteristics. The evolution of QPO frequencies has been interpreted mostly either by means of the propagation of fluctuations associated with the hot inner flow (Ingram et al. 2009; Ingram \& Done 2011) or due to the oscillation of the post shock flow (Chakrabarti et al. 2008; Iyer et al. 2015a, and references therein). In addition, the nature of the spectral properties of the accretion disc around a black hole are modeled using a standard Keplerian disc (Shakura \& Sunvaev 1973) coupled with the 'Compton corona' (Svensson \& Zdziarski 1994; Done \& Kubota 2006). Usually, the 'Compton corona' comprises of a hot and dense electron cloud that reprocesses the soft photons (i.e., thermal emission) emitted from the Keplerian disc via inverse-Comptonization process to produce the high energy component of the radiation spectrum. All these studies were carried out considering a static 'Compton corona' Tanaka \& Lewin 1995). A self-consistent attempt has been made using a dynamical two component advective flow model
Chakrabarti \& Titarchuk 1995; Chakrabarti \& Mandal 2006) while explaining the evolution of the spectral as well as the temporal behaviour of the outbursting GBH sources (Iver et al. 2015a; Debnath et al. 2016). Recently, Poutanen et al. (2017) reported a truncated disc geometry where a disc and hot plasma coexists. But, the two component advective flow model deals with a more general scenario where the accretion disc is having two components, a sub-Keplerian halo along with a standard Keplerian disc and the central hot corona can be produced from the sub-Keplerian halo component (Giri \& Chakrabarti 2013).

In order to examine the hysteresis behavior of the outbursting sources, Meyer-Hofmeister et al. (2009) studied the q-diagram considering the advection dominated accretion flow (ADAF) model, whereas Mandal \& Chakrabarti (2010) investigated the same using two-component advective flow model. All these studies were performed without taking care of self-consistent modeling of both spectral and temporal features related to q-diagram. et nala lition, the disc-jet coupling associated with the q-diagram, mostly observed in soft intermediate state (SIMS), were also ignored. Meanwhile, in the context of the disc-jet connection, efforts were made to estimate the jet kinetic power for several GBHs considering the phenomenological models (Fender et al. 2009). Eventually, there appears contradictory claims on the role of black hole spin in powering the jets (Fender et al. 2010; Steiner et al. 2013, and references therein), although the launching of jets seems to be viable irrespective to the black hole spin parameter (Aktar et al. 2015; Chattopadhyay \& Kumar 2016). Overall, a complete investigation considering the evolution of the broadband energy spectra, QPO frequencies and spectral signature of disc-jet connection for the outbursting black hole sources still remains a task to be undertaken.

Motivating with this, in the present work, we model the broadband energy $(3-150 \mathrm{keV}$ ) spectra (span over $\sim 166$ days) of XTE J1859+226 during 1999 outburst considering two-component advective flow model around a black hole. Here, we examine the overall hysteresis behaviour (in HID) of the source considering the accretion-ejection mechanism. While doing this, we theoretically compute the kinetic jet power corresponding to a radio flare (observed as jet on MJD 51479.94) and find that the calculated mass loss is in agreement with the change in accretion rate required to model the broadband spectral data. Moreover, we model the evolution of QPO frequencies while estimating the size of the post-shock region which is consistent with the best fit spectral parameters (namely, shock location, see $\S 3.3)$. Finally, based on our recently developed broadband spectral model (Iver et al. 2015a), we constrain the mass of the black hole source under consideration. 
In the next section, we describe our model along with the details of observation and analysis of the source XTE J1859+226 during 1999 outburst. In $\S 3$, we present the results and estimate the mass of the source. In $\S 4$, we discuss our results and finally present conclusion.

\section{Model and Observation}

\subsection{Model Description}

We consider a geometrically thin accretion disc around a Schwarzschild black hole. The disc is assumed to be comprised of two-component advective flows where high angular momentum viscous Keplerian flow (Shakura \& Sunyaev 1973) resides at the disc equatorial plane and is flanked by the low angular momentum weakly viscous sub-Keplerian flow (Chakrabarti \& Titarchuk 1995; Wu et al. 2002; Smith ett 2007). In order to satisfy the inner boundary conditions imposed by the black hole horizon, the accreting flow must be transonic in nature Abramowicz \& Zurek 1981). Hence, the adopted 'hybrid' accretion flow must coalesce together to become sub-Keplerian before entering onto the black hole (Giri \& Chakrabarti 2013). The radial velocity of a Keplerian flow always remain subsonic, whereas depending on the input parameters, a sub-Keplerian accretion flow may contain multiple sonic points (Chakrabarti 1989). Overall, the subsonic flow at the outer edge of the disc starts accreting towards the black hole due to gravity and becomes supersonic after crossing the outer sonic point (usually forms far away from the black hole; Das et al. (2001)). Rotating flow continues its journey further towards the horizon although a virtual barrier around the black hole is developed due to the centrifugal repulsion that finally triggers a discontinuous transition of the flow in the form of a shock wave (Fukue 1987; Chakrabarti 1989). At the shock, pre-shock supersonic flow jumps to subsonic branch where the flow kinetic energy is converted into thermal energy. Hence, due to shock, post-shock flow becomes hot and dense compared to the pre-shock flow and it behaves like a 'Compton cloud', which we refer as post-shock corona (hereafter, PSC). Finally, the subsonic post-shock flow becomes supersonic after passing through the inner sonic point and eventually enters into black hole. We assume that the Keplerian flow terminates at the shock and the PSC is fully sub-Keplerian in nature. The soft photons from the optically thick Keplerian disc are intercepted by the optically thin PSC and inverse-Comptonized to produce hard Xray power-law distribution Chakrabarti \& Titarchuk
1995; Chakrabarti \& Mandal 2006, and reference therein). We calculate the radiation spectrum from both Keplerian and sub-Keplerian components of the accretion flow to model the observed broadband radiation spectra (Iver et al. 2015a). Interestingly, PSC may oscillate due to resonance oscillation (Molteni et al. 1996) and/or for a critical viscosity that perturbs the shocked flow (Lee et al. 2011; Das et al. 2014) and exhibits QPOs. Finally, following the prescription of Chakrabarti \& Manickam (2000), in this work, the evolution of QPO frequencies in GBH sources is modeled based on the propagatory oscillating shock solution (POS) Chakrabarti et al. 2008; Chakrabarti. Dutta. \& Pal 2009; Nandi et al. 2012).

In order to describe the space-time geometry around a Schwarzchild black hole, here we adopt a pseudoNewtonian potential given as $\Phi(r)=-1 /(r-1)$ (Paczyński \& Wiita 1980). This pseudo potential allows us to solve the governing flow equations following t twe Tewtonian approach while retaining all the salient features of strong gravity. Here, $r$ denotes the radial distance measured in units of Schwarzchild radius, $r_{g}=2 G M_{b h} / c^{2}$ where $G, M_{b h}$ and $c$ are the gravitational constant, black hole mass and speed of light, respectively.

\subsection{Salient features of XTE J1859+226}

We select the Galactic black hole source XTE J1859 +226 , which has undergone outburst phase only during 1999 in the entire RXTE era. The coordinated RXTE campaign of the source enables us to study the HID profile which exhibits spectral state transitions and displays the presence of different types of QPOs Casella et al. 2004; Homan \& Belloni 2005; Radhika \& Nandi 2014, and references therein). During the same outburst, the source has been observed with multiple radio jet ejections which are relativistic in nature (Brocksopp et al. 2002).

An orbital period of $6.58 \pm 0.05 \mathrm{~h}$ and radial velocity amplitude of $541 \pm 70 \mathrm{~km} \mathrm{sec}{ }^{-1}$ have been obtained based on the optical photometry and spectroscopy of the binary system. Based on this, the mass function of the companion is obtained as $4.5 \pm 0.6 M_{\odot}$, and for an assumed inclination angle $70^{\circ}$, the lower limit of the dynamical mass of this source has been estimated as $5.42 M_{\odot}$ (Corral-Santana et al. 2011). The previous estimate of the source mass was reported to be $7.7 \pm$ $1.2 \mathrm{M}_{\odot}$ (Shaposhnikov \& Titarchuk 2009). Hence, it is clear that there are uncertainties in the estimate of the source mass. This encourages us to study the spectral, temporal and ejection characteristics of the source XTE $\mathrm{J} 1859+226$ and subsequently we constrain the mass of the source. 


\subsection{Observation and Analysis}

We analyze the public archival data of RXTE satellite for the 1999 outburst of XTE J1859+226. Pointed observations since October 9, 1999 to March 23, 2000 that span over $\sim 166$ days have been considered for the present work.

Spectral and temporal analysis are carried out using PCA and HEXTE data in 3-150 keV energy band. We follow the standard data reduction procedures as discussed in detail in Casella et al. (2004); Nandi et al. (2012); Radhika et al. (2016). For temporal analysis, we compute the power spectra of all data sets in the rising phase of the outburst. A combination of Lorentzian features are used for the modeling of the power spectra in order to find out the centroid frequencies of QPOs (Casella et al. 2004) which display a monotonic increase in frequency during the rising phase of the outburst. These QPOs are of C-type in nature and are used to model the evolution of QPOs which is presented in $\S 3.5$. We model the broadband energy spectra (PCA: $3-20 \mathrm{keV}$ and HEXTE: $20-150 \mathrm{keV}$ ) using phenomenological models consisting of diskbb and powerlaw components. The fluorescent Fe line at $6.4 \mathrm{keV}$ is modeled using a Gaussian of width $0.7 \mathrm{keV}$. From the phenomenological spectral modeling, we estimate the 'unabsorbed' flux (in units of $10^{-9} \mathrm{erg} \mathrm{cm}^{-2} \mathrm{~s}^{-1}$ ) in $3-6 \mathrm{keV}, 6-20 \mathrm{keV}$ and $3-20 \mathrm{keV}$ energy bands for generating the HID as shown in Fig. 4a (marked as blue stars). Finally, we model the broadband energy spectra based on two-component flow model (see §3.1) and compute the flux $(3-20 \mathrm{keV})$ and hardness ratio (HR: ratio of flux in $6-20 \mathrm{keV}$ to $3-6 \mathrm{keV}$ ) to reproduce the model HID profile.

\section{Modeling and Results}

We model the broadband energy spectra in the energy band of $3-150 \mathrm{keV}$ using two-component advective flow model scheme implemented in XSPEC (Iyer et al. 2015a). We estimate the modeled flux in $3-6 \mathrm{keV}$, $6-20 \mathrm{keV}$ and $3-20 \mathrm{keV}$ bands to reproduce the ' $\mathrm{q}$ diagram' for the 1999 outburst of XTE J1859+226. We also study the spectral signature of jets, using broadband spectral modeling before and after the detection of the radio flare. We calculate the jet kinetic power and mass outflow rate for an advective sub-Keplerian disc (Aktar et al. 2015; Nandi et al. 2015). In addition, we model the evolution of QPO frequencies in the rising phase of the outburst (i.e., in LHS \& HIMS), which generally are of C-type QPOs.

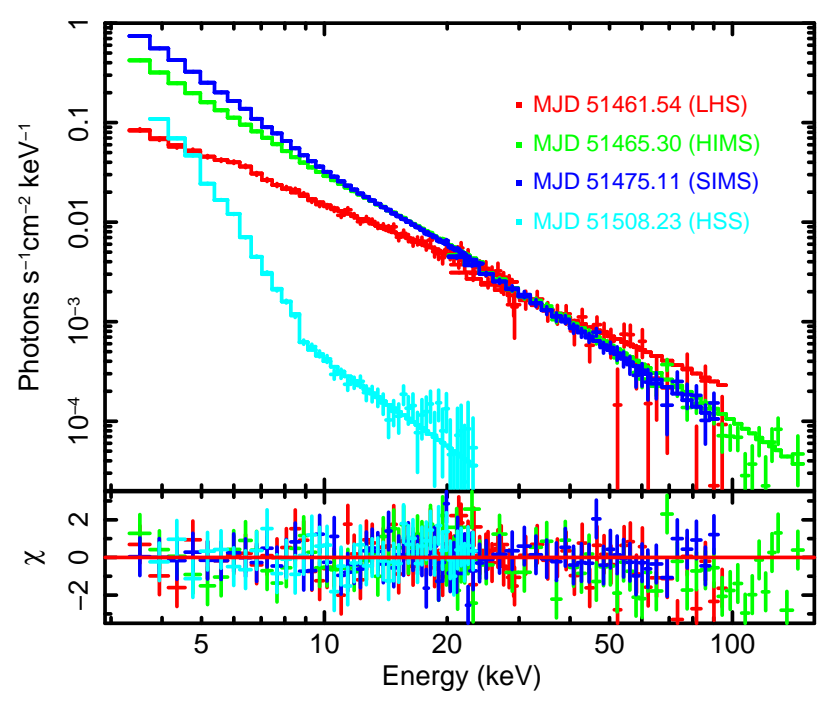

Fig. 1 Broadband energy spectra $(3-150 \mathrm{keV})$ using PCA and HEXTE data of RXTE fitted by two-component flow model for different states (LHS, HIMS, SIMS and HSS). Residuals are shown in bottom panel. See text for details.

\subsection{Modeling of Broadband Energy Spectra}

In two-component advective flow model Chakrabarti \& Titarchuk 1995; Chakrabarti \& Mandal 2006), we solve the hydrodynamic equations in presence of dissipative processes (radiative cooling) for the sub-Keplerian component of the flow and treat the Keplerian disc as the source of soft blackbody photons only. We calculate the energy density of intercepted soft blackbody photons at PSC from every annuli of the Keplerian disc. The intercepted photon energy density is used to calculate the cooling efficiency due to inverse-Comptonization in the PSC. Moreover, for a set of flow input parameters, we obtain the flow variables, namely the number density, electron temperature and proton temperature, by solving the governing hydrodynamic equations (Mandal \& Chakrabarti 2005). Subsequently, these flow variables are employed to calculate the accretion disc radiation spectrum.

We have implemented the above model in $X S P E C$ (Iyer et al. 2015a) with four input parameters, namely black hole mass $\left(M_{b h}\right.$, in units of $\left.M_{\odot}\right)$, shock location $\left(r_{s}\right.$, in units of $\left.r_{g}\right)$, Keplerian $\left(\dot{m}_{d}\right)$ and subKeplerian $\left(\dot{m}_{h}\right)$ mass accretion rates (in units of Eddington rate). Here, the soft photons (controlled by $\dot{m}_{d}$ ) are supplied by the Keplerian disc and the high energy part of the spectrum is generated due to the inverse-Comptonization of these soft photons by hot electrons in the PSC which is determined by $r_{s}$ and $\dot{m}_{h}$. In this model, the high energy and low energy part of the radiation spectrum are not independent 
Table 1 Best fit parameters required to model the broadband energy spectra $(3-150 \mathrm{keV})$ in various spectral states

\begin{tabular}{|c|c|c|c|c|c|c|}
\hline Day & State & $\begin{array}{c}\text { Shock location } \\
\left(r_{s}\right)\end{array}$ & $\begin{array}{c}\text { Sub-Keplerian } \\
\text { rate, } \dot{m}_{h}\left(\dot{M}_{E d d}\right)\end{array}$ & $\begin{array}{c}\text { Keplerian } \\
\text { rate, } \dot{m}_{d}\left(\dot{M}_{E d d}\right)\end{array}$ & $\begin{array}{c}\text { Mass } \\
\left(M_{\odot}\right)\end{array}$ & $\chi^{2} /$ dof \\
\hline 1 & LHS & $151.0 \pm 28.2$ & $0.310 \pm 0.003$ & $0.125 \pm 0.008$ & $5.79 \pm 0.24$ & $98.85 / 76$ \\
5.3 & HIMS & $36.5 \pm 3.0$ & $0.135 \pm 0.001$ & $1.630 \pm 0.150$ & $5.70 \pm 0.13$ & $117.57 / 79$ \\
15.4 & SIMS & $20.0 \pm 0.4$ & $0.142 \pm 0.002$ & $1.900 \pm 0.058$ & $6.00 \pm 0.11$ & $46.48 / 65$ \\
48 & HSS & $5.5 \pm 0.5$ & $0.048 \pm 0.001$ & $0.390 \pm 0.030$ & $5.90 \pm 0.45$ & $32.85 / 39$ \\
\hline
\end{tabular}

rather determined by the flow hydrodynamics and coupled through the flow input parameters.

During the 1999 outburst of XTE J1859+226, the source evolved from hard to soft state via intermediate states, and finally decays to the hard state Casella et al. 2004; Radhika \& Nandi 2014). We fit the broadband spectra $(3-150 \mathrm{keV})$ for the entire outburst (whenever data is available). In Fig. 1, we show the broadband model fitting of: hard state (LHS by red curve) observed on MJD 51461.54, hard intermediate (HIMS by green curve) on MJD 51465.30, soft intermediate (SIMS by blue curve) on MJD 51475.11 and soft state (HSS by cyan curve) on MJD 51508.23. We use phabs to model the inter-stellar extinction. Moreover, we also use smeared edge (smedge) and Gaussian to model intrinsic absorption and iron line signatures respectively to obtain the best fit of spectral parameters, whenever required. In the fitting, we keep a fixed hydrogen column density, $n_{H}=0.2 \times 10^{22}$ atoms $\mathrm{cm}^{-2}$ and Gaussian line energy $\sim 6.4 \mathrm{keV}$ (Radhika \& Nandi 2014, and references therein). The residuals of the model fitted spectra are shown in the bottom panel of Fig. 1 with $\chi^{2} /$ dof as 98.85/76 (LHS), 117.57/79 (HIMS), 46.48/65 (SIMS) and 32.85/39 (HSS), respectively. Best fit parameters required to model the broadband energy spectra in different spectral states (days) are presented in Table 1.

The overall spectrum is the combination of the soft and hard components and the relative normalization is determined by the fraction of soft photons intercepted by the PSC. The overall normalization is dependent on the source inclination angle and distance and it must be a constant for a given source. The expression for the norm value is proportional to $\cos \theta / D^{2}$ where $\mathrm{D}$ is in units of $10 \mathrm{kpc}$ and $\theta$ is the angle of inclination of the system. We fix the normalization corresponding to the source to estimate the mass of the source using two-component flow model fitting and adopt the following methodology to obtain the normalization value for the source. First, we fit the source spectrum from different states considering the norm as a free parameter. In reality, a physical system is more complex than an ideal one and hence normalization varies among the data sets. But, the obtained normalization values from various data sets do not vary dramatically. Hence, our best approximation of the norm value is the average of these individual norm values. Finally, we refit all the data with the average norm value to get the individual mass estimations and combine them to obtain the better mass estimate.

The broadband modeling shows (Fig. 1) that low energy photon flux increases and spectral state becomes steeper as the source transits into the soft state. The low energy flux in HSS is relatively low as the source has entered to the decay phase of the outburst (see Fig. 5). Finally, in high soft state (HSS), the high energy contribution becomes very weak as the soft photons from the Keplerian disc are able to completely cool the hot electrons in PSC.

\subsection{Confidence Intervals to Estimate the Source Mass}

As noted in the previous section, we have multiple observations of the source in different spectral states and the spectrum of each observation is being modeled independently. The mass of the central object is a fit parameter which is estimated from the spectral fit. Thus, we have multiple estimates of the mass of the central object, which can be treated as independent measurements to be combined to place a better constraint on the mass of the source. Recently, Iyer et al. (2015a) estimated the mass of black hole from broadband spectral modeling of the X-ray spectrum. We follow the same procedure in order to combine the individual mass measurement and estimate a confidence interval.

The basic premise for combining mass estimates from individual estimates comes from the application of naive Bayes theorem. In order to do this, the probability density function (PDF) of the mass value from each observation is required. To find the PDF of the mass value, we use the variation in the fit parameter $\left(\chi^{2}\right)$ about the minima of the fit along with variation in the mass value. This can be obtained using steppar command of XSPEC and it gives the confidence value $\left(C_{v_{1}}^{v_{2}}\right)$ that the mass lies in a given interval $\left(v_{1}, v_{2}\right)$ for a given change in $\chi^{2}\left(\Delta_{1}\right)$. By evaluating these confidence intervals for different values of $\Delta$, we can numerically obtain the cumulative distribution function (CDF 


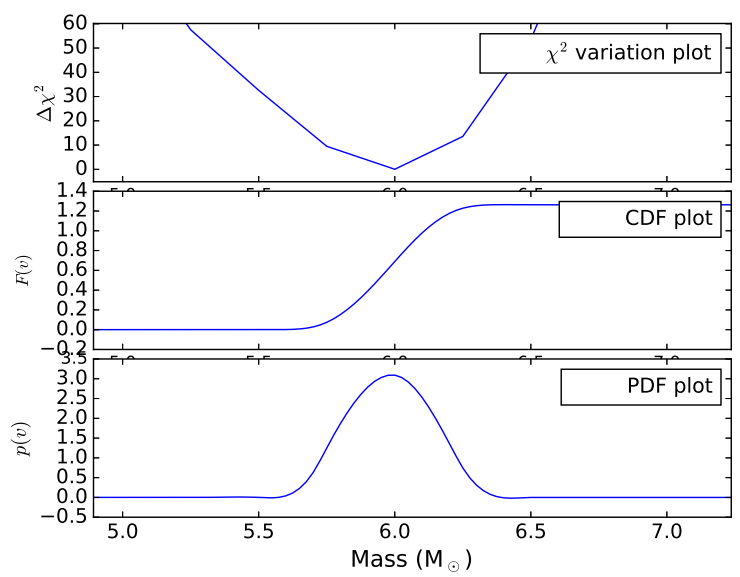

Fig. 2 Variation of $\Delta \chi^{2}, \mathrm{CDF}$ and PDF as function of mass obtained from the spectral fit of SIMS state. The $\chi^{2}$ variations about the parameter minima are used to obtain confidence intervals from which the CDF and PDF are derived. See text for details.

$=F(v)$ ) of the mass (Iyer et al. 2015a, b). From the CDF, we can then obtain the PDF $(p(v))$ of the mass. These steps are listed in equations $1-3$ and illustrated in Fig. 2 for the SIMS spectrum of XTE J1859+226.

$$
\begin{aligned}
& C_{v_{1}}^{v_{2}}=\int_{v_{1}}^{v_{2}} p(v) d v=P\left(\chi^{2}>\Delta_{1}\right), \\
& A_{v_{1}}^{v_{\text {min }}}=\frac{v_{\text {min }}-v_{1}}{v_{2}-v_{1}} C_{v_{1}}^{v_{2}} ; A_{v_{\text {min }}}^{v_{2}}=\frac{v_{2}-v_{\min }}{v_{2}-v_{1}} C_{v_{1}}^{v_{2}} \text {, } \\
& F(v)=\int_{-\infty}^{v} p(v) d v= \begin{cases}A_{-\infty}^{v_{\text {min }}}-A_{v}^{v_{\text {min }}}, & v<v_{\text {min }} \\
A_{-\infty}^{v_{\text {min }}}+A_{v_{\text {min }}}^{v}, & v>v_{\text {min }} .\end{cases}
\end{aligned}
$$

Combining these independent PDFs (obtained from fitting of different spectral states data, see Fig. 1), we then estimate an overall bound on the value of mass. This is done by using a naive Bayes approach of multiplying the individual PDFs to obtain the joint PDF which is the intersection of individual PDFs. We also obtain an estimate of the worst case upper and lower bound on the mass value by calculating the Union of PDFs as is enumerated in Iver et al. (2015b). The final mass bounds is then stated as the $90 \%$ confidence limits on each of these approaches, with the intersection based on PDF giving a tighter constrained mass value while the union PDF giving the worst case bounds. The intersection of PDFs from different data sets put a tight bound on mass but it may not particularly favour any of the PDFs. For example, if two PDFs are well separated, still their intersection may provide a narrow PDF but this estimate may not be particularly meaningful. On the other hand, if we add the PDFs, it provides an overall minimum bound, but the estimate can be poor due

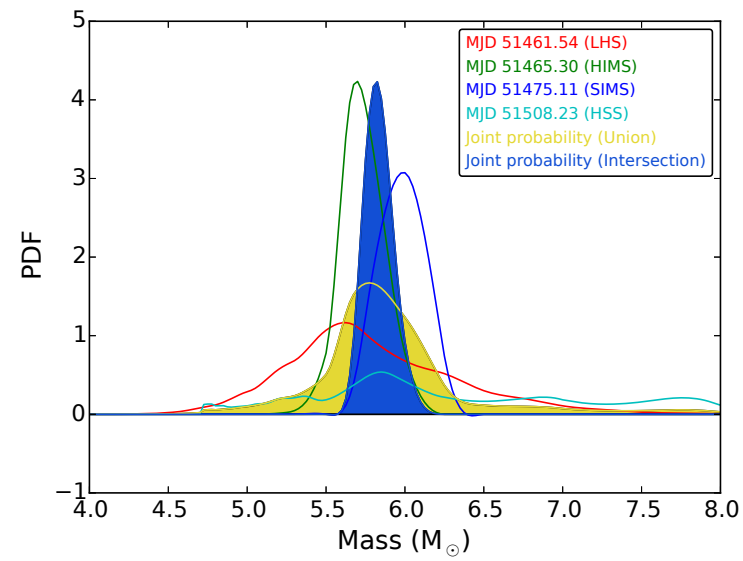

Fig. 3 PDFs obtained from individual observations of different spectral states (same with Fig. 1) as marked in the plot. Probable mass range for final Union and Intersection PDFs are shown as shaded regions. See text for details.

to systematics. This approach gives a worst case mass estimate of the source XTE J1859+226 as $5.2-7.9 M_{\odot}$ with $90 \%$ confidence as shown in Fig. 3 (yellow shaded regions) along-with the individual PDFs as obtained from various spectral states (marked in the plot). A more tighter mass constraint comes in a narrow range of $5.7-6.0 M_{\odot}$. The tighter bounds can be considered true if there are no systematic errors in the evaluation of mass from any one of the spectral states. However, with many unknown systematics being present both in the model, and in the data and uncertainties in the response obtained from RXTE, we stick to quote the worst case bounds as our final limit as $5.2-7.9 M_{\odot}$.

A lower value of mass bounds can be obtained if additional physical processes like effects of atomic lines, intrinsic disc absorptions/reflections and jet contributions are included in the model self-consistently. With this, such a model would be able to consistently fit all observations having single norm. Also, to avoid the systematics in the data, one would not be able to combine the results from all the states. Rather we may need to consider only LHS and HSS where the systematics due to atomic line emissions and jet ejections are comparatively less. Even in LHS there can be contribution due to steady winds if not jet. Other possibility of improvement is that a simultaneous multi-wavelength observations are required to model the jet/wind contributions and accordingly this contribution can be removed from the accretion disc.

Further, we attempt to get a mass bound by choosing spectra from LHS and HSS where the systematics in the data are low and modeling them simultaneously with tied mass and norm. Fig. 4 shows the simultaneous fitting of LHS and HSS spectra of XTE J1859+226 
during its 1999 outburst. The fit results give a mass of $5.91 \pm 0.28 M_{\odot}$ with $\chi^{2} /$ dof equal to $150.82 / 104$. This corresponds to an overall uncertainty of $9.46 \%$. This estimate is within the broad range given by the union of PDFs and is overlapping with the tighter bounds given by the intersection of PDFs. As the intersection of PDFs may not favor the maxima of individual PDFs, we consider the estimate based on the simultaneous fitting with tied norm and mass values from LHS and HSS.

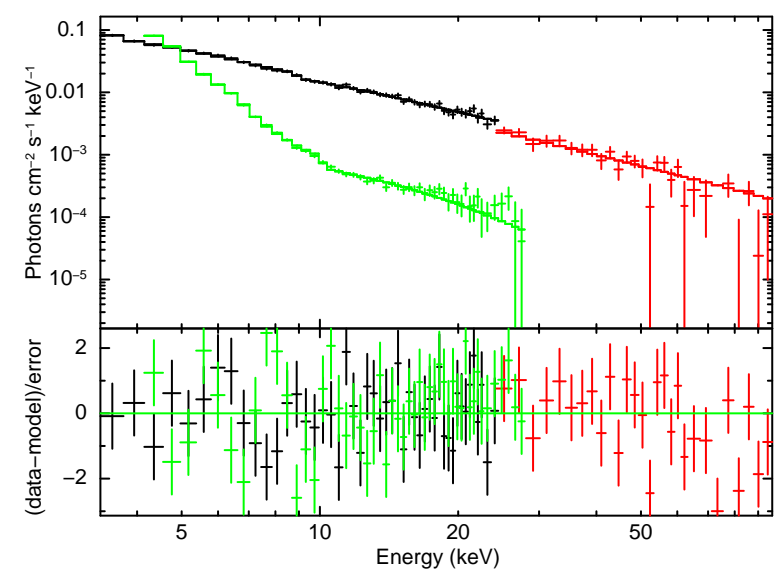

Fig. 4 Simultaneous fitting of LHS (black-red for PCA and HEXTE data) and HSS (green for PCA data) spectra from the 1999 outburst of XTE J1859+226 done by tying the mass and norm parameters. Fitted mass is around $5.91 \pm$ $0.28 M_{\odot}$, which is within the broad range of mass estimated from union of PDFs (see Fig. 3).

\subsection{Modeling of HID (q-diagram)}

In order to model the HID, we fit the entire outburst (span over $\sim 166$ days) spectral data using two-component advective flow model and compute the fluxes in the energy band of $3-20 \mathrm{keV}, 3-6 \mathrm{keV}$ and $6-20 \mathrm{keV}$. Upon estimating the fluxes and hardness ratios, we plot the variation of total flux as function of hardness ratio as shown in Fig. 5a (marked with red circles). It is to be noted that the 'modeled' profile (red filled circles) is in close agreement with the observed (blue stars) HID obtained from the phenomenological modeling of the data. We also estimate the model parameters $\left(\dot{m}_{h}, \dot{m}_{d}\right.$ and $\left.r_{s}\right)$ from the spectral fitting during the entire 1999 outburst and in Fig. 5b, we present the variation of $\dot{m}_{h}$ (green filled asterisks), $\dot{m}_{d}$ (red filled diamonds) and $r_{s}$ (blue filled circles), respectively. We also plot the total flux observed (light gray) in the same figure. It is evident that total flux increases with the total accretion rate and follows the same trend of accretion rate during the entire outburst. We observe that as the source moves from hard to soft state in the rising phase, $\dot{m}_{d}$ (red filled diamonds) increases and $r_{s}$ (blue filled circles) moves towards the black hole. While in the decay phase, the Keplerian accretion rate $\left(\dot{m}_{d}\right)$ decreases and shock $\left(r_{s}\right)$ moves outward. During the outburst, we see an anti-correlation between the two accretion rates which is expected. Except the first few days and last few days of the outburst, $r_{s}$ varies between few to $30 r_{g}$ and sub-Keplerian rate $\left(\dot{m}_{h}\right)$ remains $\sim 0.1$ and hence the outburst dynamics is mostly controlled by Keplerian accretion rate $\dot{m}_{d}$. Usually, the dynamics of the Keplerian disc is governed by viscous timescale which is typically around few days (Mandal \& Chakrabarti 2010) for a disc around a stellar mass black hole and this time scale is consistent with the rising phase ( $\sim 7-8$ days) of the outburst of XTE $\mathrm{J} 1859+226$. After the rising phase, the source continues to move towards the soft state where the supply of Keplerian matter becomes significant. Finally, as the source enters into the decaying phase of the outburst, supply of sub-Keplerian matter is increased reducing the Keplerian matter supply.

\subsection{Spectral Signature of Disc-Jet Connection and Estimation of Jet Power}

Multiple transient jets (三 radio flares) are observed in the 1999 outburst of XTE J1859+226 (Brocksopp et al. 2002) although QPOs are not seen in X-ray data (Radhika \& Nandi 2014) during the radio flares. A general understanding towards this is that multiple transient jets are observed in the intermediate state of the outbursting sources and these ejections may be triggered during the state transition from HIMS to SIMS. In this work, as a representative case, we choose the third radio flare ( $\mathrm{read}$ as $F_{3}$ ) when the source is clearly in the SIMS state. We use the X-ray spectral data during $F_{3}$ flare in order to investigate the spectral signature of the jet ejections. We model three broadband spectra before (MJD 51478.78 in red), after (MJD 51483.11 in blue) and during (MJD 51480.04 in green) the radio flare (Brocksopp et al. 2002; Radhika \& Nandi 2014) as depicted in Fig. $6\left(F_{3}\right.$ is marked in Fig. 5a and $5 \mathrm{~b}$ as well). The spectrum (green curve) during the flare indicates the presence of excess soft photon flux whereas the hard X-ray flux is noticeably reduced compared with the other two spectra (red and blue curves). During the flare, a two-component broadband spectral modeling of the observed data requires an inward movement of shock (smaller PSC) and the increase of mass accretion rate $(\sim 14 \%)$ in comparison with the same needed to model the spectral data before the flare. In Table 2, we have summarize the model fitted spectral parameters - both accretion rates, shock location and 

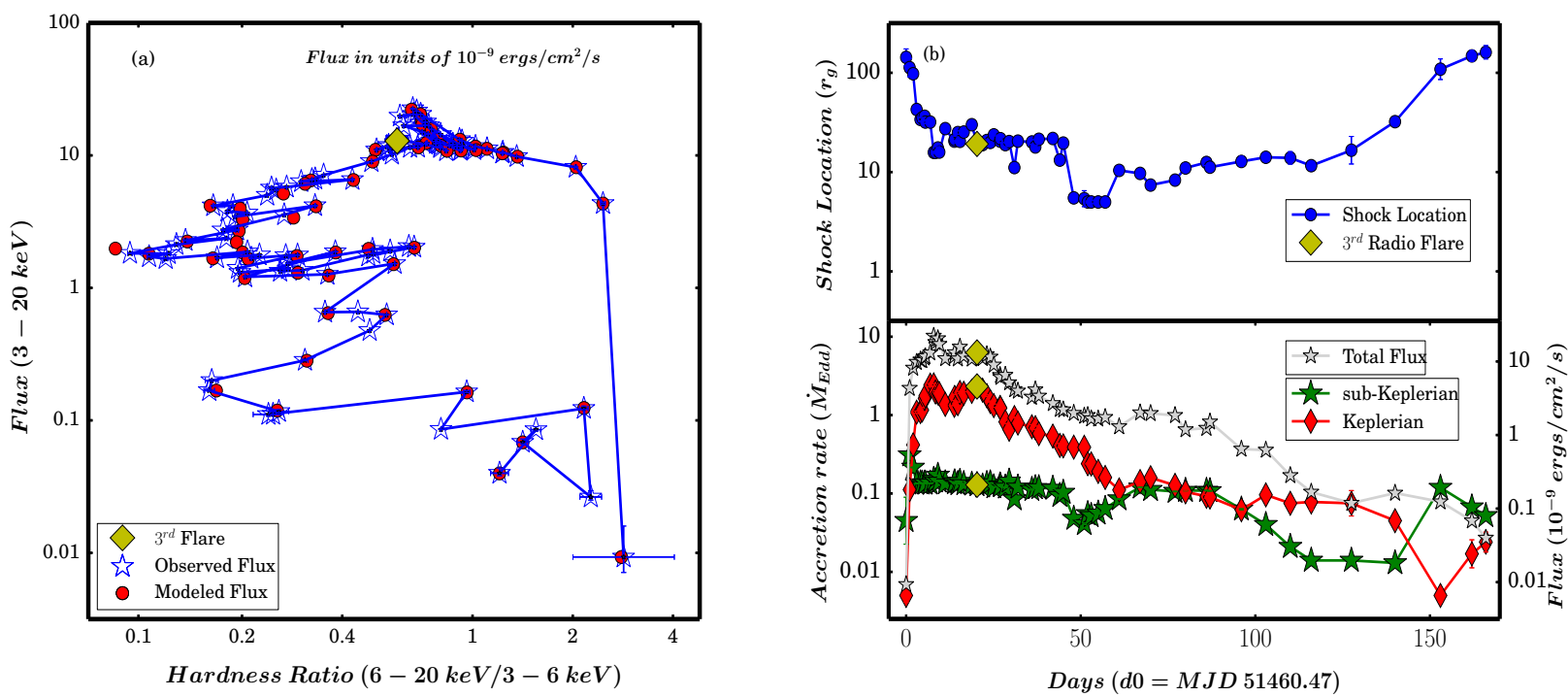

Fig. 5 (a) HID of the 1999 outburst of XTE J1859+226 plotted using X-ray flux from phenomenological model (marked with blue star along-with error bars) and two-component model based HID (marked as red filled-circle) are presented. A good agreement is seen between the modeled q-profile and the observed q-profile. (b) The time evolution of shock location $\left(r_{s}\right.$, blue circles in upper panel), sub-Keplerian accretion rate $\left(\dot{m}_{h}\right.$, green asterisks $)$, Keplerian accretion rate $\left(\dot{m}_{d}\right.$, red diamonds), and total observed flux (light gray stars) along the q-track are shown (in bottom panel). See text for details.

$\%$ change in total rates during the $\mathrm{F} 3$ radio flare. It is evident that the halo accretion rate remains almost constant across the flare and the significant change in mass accretion rate is due to the variation in Keplerian disc rate.

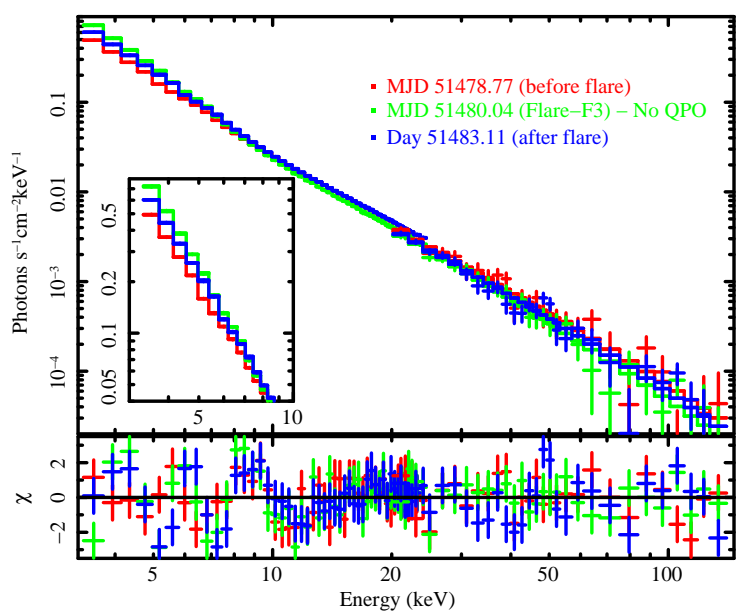

Fig. 6 Spectral signature of mass-loss during third flare $\left(F_{3}\right)$ of the 1999 outburst of XTE J1859+226. QPO frequency is not observed during the flare. Maximum change in accretion rate is observed to be $\sim 14 \%$ between before and during the radio flare. See text for details.

In order to quantify the mass flux of the outflowing matter, we calculate the observed kinetic jet power $\left(L_{\mathrm{Jet}}^{\mathrm{obs}}\right)$ employing the expression given by Longair
2011),

$$
\begin{gathered}
L_{\text {jet }}^{\mathrm{kin}} \sim 3 \times 10^{33} \eta_{\mathrm{jet}}^{4 / 7}\left(\frac{\Delta t}{\mathrm{sec}}\right)^{2 / 7}\left(\frac{\nu}{\mathrm{GHz}}\right)^{2 / 7} \\
\times\left(\frac{S_{\nu}}{\mathrm{mJy}}\right)^{4 / 7}\left(\frac{D}{\mathrm{kpc}}\right)^{8 / 7} \operatorname{erg~sec}^{-1}
\end{gathered}
$$

where $\eta_{\text {jet }}$ is the jet efficiency factor and its value is of the order of unity as estimated from observation (Fender 2001). Here, $\Delta t$ is the rise time of the jet which we consider as 24 hours as a representative value. The observed radio flux is denoted by $S_{\nu}$, where $\nu$ is the frequency of radio observation and $D$ is the distance to the source. During the $\mathrm{F}_{3}$ flare, the radio flux is observed as $S_{\nu} \sim 25$ mJy at $\nu=3.9 \mathrm{GHz}$ (Brocksopp et al. 2002). When jet moves with speed comparable to the speed of light $(c)$, emitted radiations from the jet are expected to be relativistically Doppler boosted towards the observer. In order to account the relativistic Doppler effect, we introduce a correction factor $f(\Gamma, \theta)$ while estimating the observed kinetic jet power (Fender 2001) as,

$$
L_{\text {jet }}^{\text {obs }}=f(\Gamma, \theta) \times L_{\text {jet }}^{\text {kin }} \operatorname{erg~} \sec ^{-1},
$$

where $\Gamma$ is the bulk Lorentz factor and $\theta$ is the inclination angle between the line of sight and the direction of the jet axis. In this study, we consider the inclination angle as $\theta \sim 60^{\circ}-70^{\circ}$ (Corral-Santana et al. 2011) and 
Table 2 Model fitted spectral parameters around the F3 radio flare.

\begin{tabular}{lccccr}
\hline MJD & Shock location $\left(r_{s}\right)$ & Halo rate $\left(\dot{M}_{E d d}\right)$ & Disc rate $\left(\dot{M}_{E d d}\right)$ & Total rate $\left(\dot{M}_{E d d}\right)$ & Change $(\%)$ \\
\hline 51478.77 & $30.0 \pm 0.9$ & $0.131 \pm 0.003$ & $2.01 \pm 0.072$ & $2.14 \pm 0.072$ & - \\
51480.04 (flare) & $19.2 \pm 0.6$ & $0.129 \pm 0.001$ & $2.32 \pm 0.098$ & $2.44 \pm 0.098$ & 14 \\
51483.11 & $24.8 \pm 0.8$ & $0.132 \pm 0.002$ & $2.20 \pm 0.060$ & $2.33 \pm 0.060$ & 09 \\
\hline
\end{tabular}

the bulk Lorentz factor as $\Gamma \sim 2-3$ (as jets are assumed to be mildly relativistic), respectively. Employing these values, we estimate the mean value of $f(\Gamma, \theta)=9.74$ (Fender 2001). Using the above data along with a range of distance to the source as $D=6-11 \mathrm{kpc}$ (Hvnes et al. 2002; Zurita et al. 2002), we estimate the observed kinetic jet power to be $L_{\text {jet }}^{\text {obs }} \sim 3-6 \times 10^{37}$ $\operatorname{erg~} \mathrm{s}^{-1}$.

In the context of accretion-ejection mechanism, rotating accretion flow experiences virtual barrier (i.e., PSC) in the vicinity of the black hole where a part of the accreting matter is deflected in the vertical direction to form bipolar jets (Chakrabarti 1999; Chattopadhyav \& Das 2007; Das \& Chattopadhvav 2008; Chattopadhyay \& Kumar 2016, and references therein). Towards this, Aktar et al. (2015) recently estimated the mass outflow rate $\left(R_{\dot{m}}\right)$ around the rotating black hole in terms of the inflow parameters. Moreover, using the RXTE observations of several black hole sources, they calculated the X-ray luminosity of a given source as $L_{x}=4 \pi D^{2} F_{x}$, where $F_{x}$ is the total unabsorbed X-ray flux. Following this approach and considering the radiative efficiency of the accreting matter $\eta \sim 0.06$ (for non-rotating black holes), in this work, we calculate the accretion rate of the black hole as $\dot{m}_{\text {acc }}=L_{x} / \eta c^{2}$. Subsequently, the model estimated jet kinetic power is estimated as (Aktar et al. 2015),

$$
L_{\text {jet }}^{\text {est }}=R_{\dot{m}} \times \dot{m}_{a c c} \times c^{2} \quad \operatorname{erg~sec}^{-1},
$$

where $R_{\dot{m}}$ represents the mass outflow rate defined as the ratio of outflow and inflow mass flux. In this work, using the best fit spectral parameters during flare $F_{3}$ with $r_{s} \sim 20 r_{g}$ (Fig. $5 \mathrm{~b}$ ) and the difference of $\mathrm{X}$ ray fluxes during (MJD 51480.04) and before (MJD 51478.77 ) the flare as $F_{x} \sim 2.16 \times 10^{-9} \mathrm{erg} \mathrm{cm}^{-2} \mathrm{~s}^{-1}$, we estimate the model kinetic jet power $L_{\text {jet }}^{\text {est }}$ employing equation (6). We find that $L_{\text {jet }}^{\text {est }}$ is in agreement with $L_{\text {jet }}^{\text {obs }}$ provided the mass outflow rate $\left(R_{\dot{m}}\right)$ lies in the range $\sim 8-16 \%$ of the accretion rate. This mass outflow rate is consistent with the change in accretion rate $(\sim 14 \%)$ required to fit the broadband spectra (Fig. 6$)$ before and during the jet ejection as mentioned earlier.

\subsection{Evolution of QPO Frequency $(\nu)$}

In this section, we examine the evolution of QPO frequencies during the rising phase of the outburst follow- ing the Propagating Oscillatory Shock (POS) model (Chakrabarti et al. 2008, Chakrabarti. Dutta, \& Pal 2009, Nandi et al. 2012, Iver et al. 2015a), where shock location is treated as free parameter.

The frequency of the QPO which is the rate at which the shock front oscillates is given by (Iver et al. $2015 \mathrm{a}$ and references therein), $\nu_{q p o}=\frac{c}{2 \pi R r_{g} r_{s} \sqrt{r_{s}-1}}$, where $R$ is the compression ratio defined as the ratio of postshock to the pre-shock matter densities. In this model, the mass of the black hole that appears in the expression of $r_{g}$ is also a free parameter. Due to the limited number of observed data points (see Fig. 7) as well as the limited knowledge of the methodology itself, namely how $r_{s}$ is evolving with time, here we use the black hole mass estimated from the broadband spectral modeling and consider it as a fixed parameter. In the rising phase of the outburst, the QPO frequency increases with time and we assume that the shock is moving inward with an acceleration $\left(a_{t}\right)$. Hence, the location of the shock is changing with time $(\mathrm{t})$ as $r_{s}=r_{s 0}-\left(v_{o} t+0.5 a_{t} t^{2}\right) / r_{g}$, where $r_{s 0}$ is the initial shock location and $v_{0}$ is the initial shock drift speed. These parameters are estimated from the model fitting of the evolution of QPO frequencies. The evolution of the QPO frequencies (black points) and its model fitting (magenta curve) are depicted in Fig. 7. The estimated shock locations are presented by red circles. The bottom panel shows the residual of the fitted QPO frequencies.

In Fig. 7, we over plot the shock locations (blue stars) estimated from spectral modeling, which are in close agreement (except the 1st observation) with POS model fitted shock locations. We also estimate the QPO frequencies using POS formula considering the model fitted shock locations. The calculated QPO frequencies are shown in Fig. 7 (green stars). The results are summarized in Table 3, where comparison between results obtained from temporal (POS) and spectral (twocomponent) modeling are given. Here, the best fit requires $v_{0}=20.9 \mathrm{~m} \mathrm{~s}^{-1}, r_{s 0}=263 r_{g}$ respectively. According to our analysis, initially the oscillating shock front accelerates inward with $a_{t} \sim-1.08 \mathrm{~m} \mathrm{~s}^{-1} \mathrm{day}^{-1}$ up to the shaded region (both observations show similar spectro-temporal features) which is followed by an acceleration $a_{t} \sim-5.08 \mathrm{~m} \mathrm{~s}^{-1} \mathrm{day}^{-1}$ in the HIMS. The estimated shock locations (i.e., size of PSC) decreases as the QPO frequencies (C-types) evolve from LHS to 
HIMS which is consistent with the variation of $r_{s}$ estimated from the broadband spectral modeling (Fig. 5b; Table 3). Since A-type and B-type QPOs observed in SIMS do not show noticeable evolution (Casella et al. 2004; Radhika \& Nandi 2014), we exclude them while modeling the evolution of QPO frequencies.

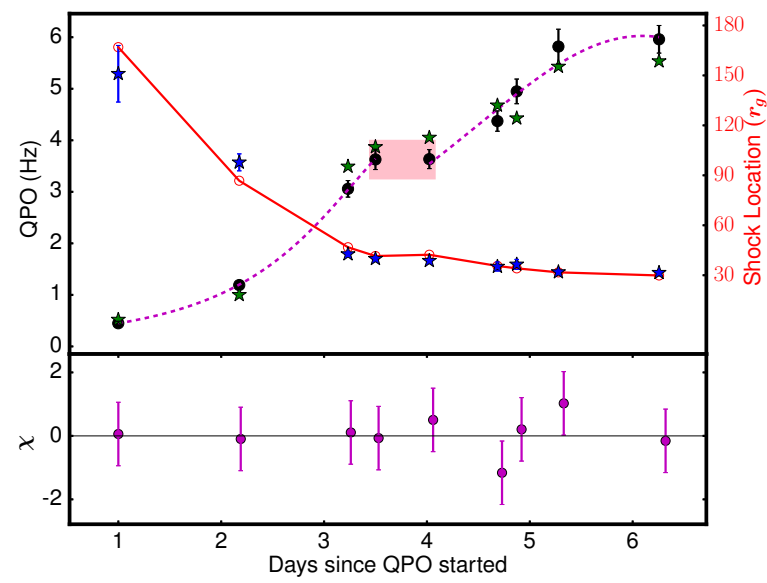

Fig. 7 Evolution of QPO frequencies (black points) in LHS and HIMS with time (days) of the 1999 outburst of XTE J1859+226 is fitted with POS model solution (dotted magenta curve). The model fitted shock locations (red curve) decreases with the increase of QPO frequencies as predicted by the model. Residuals of POS fitting are shown in bottom panel. The shock location (blue stars) estimated from two-component flow modeling of energy spectra and the corresponding estimated QPO frequencies (green stars) are also shown.

Next, we demonstrate that the two values of frequencies, measured independently from timing and spectral modeling, agree quite well. For this, we have plotted observed QPOs against $1 /\left(r_{s} \sqrt{\left(r_{s}-1\right)}\right)$, variable term in POS equation, where $r_{s}$ is obtained from spectral modeling. We have fitted this the variation with a straight line (Fig. 8). The slope of the fitting is 1006.43 with an interception of -0.15. Comparing with the POS formula, the value of $R * r_{g}$ is obtained as 47441.16 , which provides a compression ratio $(\mathrm{R})$ as 2.87 . The estimated $\mathrm{R}$ lies in the theoretically accepted limit $(1-4)$ for non-relativistic shocks.

\section{Discussion and Conclusions}

In this paper, we examine the accretion flow dynamics of the outbursting XTE J1859+226 source by modeling the spectral and temporal characteristics along its q-track. Towards this, we theoretically model the broadband $(3-150 \mathrm{keV})$ spectral evolution of the 1999

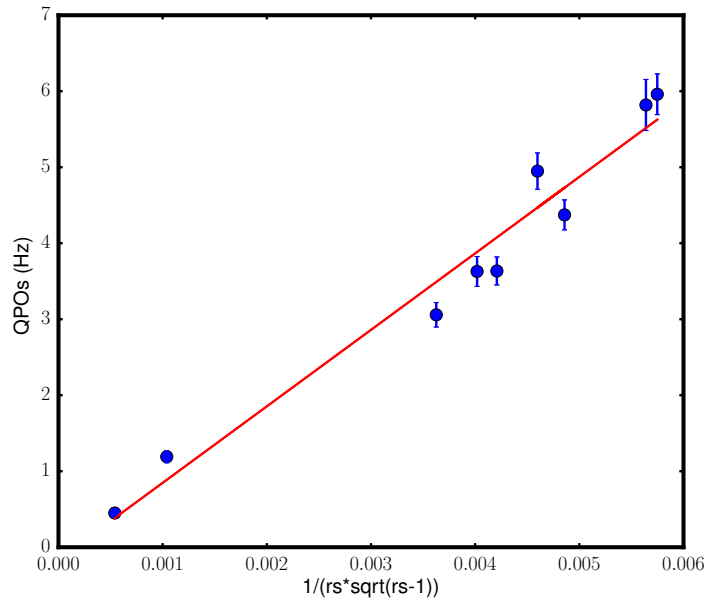

Fig. 8 Correlation between QPO frequencies and the POS model function (POS formula; §3.5) obtained from energy spectral modeling.

outburst (span over $\sim 166$ days) of this source using two-component advective flow model. In this analysis, four input parameters, namely Keplerian accretion rate $\left(\dot{m}_{d}\right)$, sub-Keplerian accretion rate $\left(\dot{m}_{h}\right)$, shock location $\left(r_{s}\right)$ and black hole mass $\left(M_{b h}\right)$, are used to model all the observations. Apart from this, the normalization parameter of the model is a constant scaling factor for a given source. We have fitted spectra from various states of the source considering norm as a free parameter and then take an average of all these independent norm values. This average norm value is used as a constant norm for all the observations of the source under consideration. The model fitted norm value provides an estimate of the product $\cos \theta / D^{2}$ and does not allow an independent estimate of distance and inclination angle of the source. If the inclination angle is assumed to lie between $60^{\circ}-70^{\circ}$ (Corral-Santana et al. 2011) for this source, the distance is calculated as $4.8-5.8 \mathrm{kpc}$ which is in agreement with the distance range $4.6-8$ kpc reported by Hynes et al. (2002).

Following our approach as mentioned in $\S 3.1$ and $\S 3.3$, we reproduce the q-profile of the source which is in agreement with the observed q-diagram (Fig. 5a). Moreover, our results suggest that during the rising phase of the outburst, Keplerian accretion rate increases and shock front moves inwards (Fig. 5b) while the sub-Keplerian accretion rate initially decreases and becomes almost constant afterwards. On the contrary, towards the end of the outburst both the accretion rates tend to readjust where sub-Keplerian counter part exceeds the Keplerian component.

In the decay phase, a reverse trend in the variation of accretion rates is seen and PSC reconciles the hysteresis 
Table 3 Comparison of QPO frequencies and shock location estimated from temporal and spectral modeling. We get $r_{s m}$ and $\nu$ by modeling energy spectra with two-component flow model and POS formula, while $r_{s o}$ is obtained from POS fitting of observed QPOs.

\begin{tabular}{l|c|c|c|c|r}
\hline MJD & QPO $(\mathrm{Hz})$ & FWHM $(\mathrm{Hz})$ & $r_{s m}\left(r_{g}\right)$ & $r_{s o}\left(r_{g}\right)$ & $\nu(\mathrm{Hz})$ \\
\hline 51461.57 & 0.45 & 0.089 & $151.0 \pm 17.0$ & 166.96 & 0.52 \\
51462.76 & 1.19 & 0.187 & $97.80 \pm 5.1$ & 86.76 & 1.00 \\
51463.83 & 3.05 & 0.378 & $42.70 \pm 1.3$ & 46.80 & 3.49 \\
51464.10 & 3.62 & 0.462 & $39.90 \pm 1.1$ & 41.52 & 3.87 \\
51464.63 & 3.63 & 0.433 & $38.70 \pm 0.8$ & 42.30 & 4.05 \\
51465.30 & 4.37 & 0.464 & $35.20 \pm 2.7$ & 35.58 & 4.67 \\
51465.49 & 4.94 & 0.562 & $36.50 \pm 3.0$ & 34.14 & 4.42 \\
51465.90 & 5.81 & 0.790 & $31.90 \pm 1.1$ & 31.73 & 5.43 \\
51466.89 & 5.96 & 0.630 & $31.50 \pm 0.9$ & 29.86 & 5.53 \\
\hline
\end{tabular}

property of this source. Also, the broadband spectral modeling indicates that as the source moves towards softer states, the low energy X-ray flux increases with a steepening of high energy power law (Fig. 1). Further, we constrain the mass of the source using broadband spectral modeling by generating PDFs (Fig. 3) obtained from different spectral states as discussed in $\S 3.2$. The mass range is estimated as $5.2-7.9 M_{\odot}$ with $90 \%$ confidence which is above the dynamically measured lower mass limit (Corral-Santana et al. 2011). We quote this larger mass range as the effect of systematics in model and data. Model systematics include effects from atomic lines, winds and jet emissions and these need to be self-consistently included in the model. To check the systematics in data, we fit LHS and HSS data considering tied norm and mass simultaneously and following this, we obtain the mass estimate as $5.91 \pm 0.28 M_{\odot}$ with an overall uncertainty of 9.46 $\%$.

During the radio flare, the reduction of hard X-ray flux is observed (green curve in Fig. 6), as a part of the accreting matter is ejected from the PSC in the form of jets (see §3.4). Hence, the size of PSC is reduced that allows the Keplerian disc to move inwards enabling the increase of Keplerian accretion rate. With this, the availability of soft photons is enhanced significantly. A few hours after the flare (blue curve in Fig. 6), shock recedes outward and the contribution of hard X-ray flux increases. A similar characteristics is also noticed during all the radio flares observed in this source (Radhika \& Nandi 2014), as well as other black hole sources (Vadawale et al. 2001; Nandi et al. 2001; Radhika et al. 2016). In addition, we compute the kinetic jet power $\left(L_{\text {jet }}^{\text {obs }}\right)$ employing the observed radio flux during the third flare and $L_{\text {jet }}^{\text {obs }}$ is then used to estimate the mass outflow rate $\left(R_{\dot{m}}\right)$ which is found to be consistent with the change in accretion rate $\left(\dot{m}_{h}+\dot{m}_{d}\right)$ required to fit the spectral data before and during the radio flare.
Employing the POS solution (§3.5), it is ascertained that the shock decelerates inward in the initial phase and further slows down to a smaller value in SIMS phase. This evidently indicates that QPO frequency increases during the rising phase of the outburst (Fig. 7). Moreover, we find that shock location calculated from the POS model is consistent with the size of the corona (i.e., PSC) as obtained from spectral modeling (Fig. 5b). In Fig 8. we show the correlation of QPO frequencies with the functional form $\left(r_{s}\right.$ estimated from spectral modelling) of the POS equation. The slope of the correlation (after fitting a straight line) provides a compression ratio $(\mathrm{R})$ of around 2.87 , well within the expected range.

Spectro-temporal variability similar to the source XTE J1859 + 226 is also observed in multiple outbursts of the source GX 339-4 (Nandi et al. 2012, and references therein) and in other outbursting GBH sources (Remillard \& McClintock 2006). The investigation of these variabilities observed in other $\mathrm{GBH}$ sources and constraining the mass of the sources are under progress and will be reported elsewhere.

\section{Acknowledgements}

Authors are thankful to the reviewer for his/her valuable suggestions and comments that helped to improve the quality of the manuscript. This research has made use of data obtained through the High Energy Astrophysics Science Archive Research Center (HEASARC) online service, provided by the NASA/Goddard Space Flight Center. AN thanks GD, SAG; DD, PDMSA and Director, ISAC for encouragement and continuous support to carry out this research.

\section{References}

Abramowicz, M. A. \& Zurek, W. H., 1981, Astrophys. J., 246,314 
Aktar, R., Das, S., \& Nandi, A., 2015, Mon. Not. R. Astron. Soc., 453, 3414

Aktar, R., Das, S., Nandi, A., \& Sreehari, H. 2017, Mon. Not. R. Astron. Soc., 471, 4806

Belloni, T., Psaltis, D., \& van der Klis, M., 2002, Astrophys. J., 572, 392

Belloni, T., Homan, J., Casella, P., et al., 2005, Astron. Astrophys., 440, 207

Brocksopp, C., et al., 2002, MNRAS, 331, 765

Casella, P., Belloni, T., Homan, J., \& Stella, L., 2004, A\&A, 426,587

Chakrabarti, S K., \& Titarchuk, L., 1995, Astrophys. J., 455,623 .

Chakrabarti, S. K., 1999, Astron. Astrophys., 351, 185

Chakrabarti, S. K., \& Manickam, S. G., 2000, Astrophys. J., 531, L41

Chakrabarti, S. K., \& Mandal, S., 2006, Astrophys. J. Lett., 642, L49

Chakrabarti, S. K., Debnath, D., Nandi, A., \& Pal, P. S., 2008, Astron. Astrophys., 489, L41

Chakrabarti, S. K., Dutta, B. G., \& Pal, P. S., 2009, MNRAS, 394, 1463

Chakrabarti, S. K., 1989, ApJ, 347, 365

Chattopadhyay, I., \& Das, S., 2007, New Astron., 12, 454

Chattopadhyay, I., \& Kumar, R. 2016, Mon. Not. R. Astron. Soc., 459, 3792

Corbel, S., \& Fender, R. P., 2002, Astrophys. J., 573, L35

Corbel, S., Nowak, M., Fender, R. P., et al., 2003, Astron. Astrophys., 400, 1007

Corbel, S., Fender, R. P., Tomsick, J. A., et al., 2004, Astrophys. J., 617, 1272

Corral-Santana, J. M., et al., 2011, Mon. Not. R. Astron. Soc., 413, 15

Das, S., Chattopadhyay, I., \& Chakrabarti, S. K., 2001, ApJ, 557, 983

Das, S., \& Chattopadhyay, I., 2008, New Astron., 549, 556

Das, S., Chattopadhyay, I., Nandi, A., \& Molteni, D., 2014, Mon. Not. R. Astron. Soc., 442, 251

Debnath, D., Chakrabarti, S. K., \& Nandi, A. 2013, Advances in Space Research, 52, 2143

Debnath, D., Chakrabarti, S.K., \& Mondal, S., 2016, Mon. Not. R. Astron. Soc., 440, 121

Done, C., \& Kubota, A., 2006, Mon. Not. R. Astron. Soc., 371,1216

Feroci, M., Matt, G., Pooley, G., et al. 1999, Astron. Astrophys., 351, 985

Fender, R. P., 2001, Mon. Not. R. Astron. Soc., 322, 31

Fender, R. P., Belloni, T., \& Gallo, E., 2004, Mon. Not. R. Astron. Soc., 355, 1105

Fender, R. P., Homan, J., \& Belloni, T., 2009, Mon. Not. R. Astron. Soc., 396, 1307

Fender, R. P., Gallo, E., \& Russell, D., 2010, Mon. Not. R. Astron. Soc., 406, 1425

Fender, R., \& Gallo, E., 2014, Space Sci. Rev., 183, 323

Fukue, J., 1987, PASJ, 39, 309

Giri, K., \& Chakrabarti, S. K., 2013, MNRAS, 430, 2836

Homan, J., \& Belloni, T., 2005, Astrophys. Space Sci., 300, 107

Hynes, R. I., Haswell, C. A., Chaty, S., Shrader, C. R. \& Cui, W., 2002, Mon. Not. R. Astron. Soc., 331, 169-179
Iyer, N., Nandi, A., \& Mandal, S., 2015a, Astrophys. J., 807,108

Iyer, N., Nandi, A., \& Mandal, S., 2015b, Astronomical Society of India Conference Series, 12, 97

Ingram, A., Done, C., \& Fragile, P. C., 2009, Mon. Not. R. Astron. Soc., 397, L101

Ingram, A., \& Done, C., 2011, Mon. Not. R. Astron. Soc., 415,2323

Longair, S. M., 2011, High Energy Astrophysics (Cambridge University Press)

Lee, S. J., Ryu, D., \& Chattopadhyay, I., 2011, Astrophys. J., 728, 142

Maccaroni, T. J., \& Coppi, P.S., 2003, Mon. Not. R. Astron. Soc., 338,189

Mandal, S., \& Chakrabarti, S. K. 2005, Astron. Astrophys., 434,839

Mandal, S., \& Chakrabarti, S. K., 2010, Astrophys. J. Lett., 710, L147

Meyer-Hofmeister, E., Liu, B. F., \& Meyer, F., 2009, Astron. Astrophys., 508, 329

Miller-Jones, J. C. A., Sivakoff, G. R., Altamirano, D., et al., 2012, Mon. Not. R. Astron. Soc., 421, 468

Mirabel, I. F., \& Rodríguez, L. F. 1994, Nature, 371, 46

Molteni, D., Sponholz, H., \& Chakrabarti, S. K., 1996, Astrophys. J., 457, 805

Nandi, A., Chakrabarti, S. K., Vadawale, S. V., \& Rao, A. R., 2001, Astron. Astrophys., 380, 245

Nandi, A., Debnath, D., Mandal, S., \& Chakrabarti, S. K., 2012, Astron. Astrophys., 542, A56

Nandi, A., Mandal, S., Das, S., \& Chattopadhyay, I., 2015, Astronomical Society of India Conference Series, 12, 69

Paczyński, B., \& Wiita, P. J., 1980, Astron. Astrophys., 88, 23.

Poutanen, J., Veledina, A., Zdziarski, A. A., 2017, arXiv 1711.08509

Radhika, D., \& Nandi, A., 2014, AdSpR, 54, 1678

Radhika, D., Nandi, A., Agrawal, V. K., \& Seetha, S., 2016, MNRAS, 460, 4403

Remillard, R. A., \& McClintock, J. E., 2006, Annu. Rev. Astron. Astrophys., 44, 49

Shakura, N. I., \& Sunyaev, R. A., 1973, Astron. Astrophys., 24,337

Shaposhnikov, N., \& Titarchuk, L., 2009, Astrophys. J., 699,453

Smith, D. M., Dawson, D. M., \& Swank, J. H., 2007, Astrophys. J., 669, 1138

Soleri, P., Belloni, T., \& Casella, P., 2008, Mon. Not. R. Astron. Soc., 383, 10989

Sreehari, H., Nandi, A., Radhika, D., Iyer, N. \& Mandal, S., J. Astrophysics. Astronomy, 39, arXiv 1802.05163

Steeghs,D., J. E. McClintock, S. G. Parsons, M. J. Reid, S. Littlefair, \& V. S. Dhillon, 2013, Astrophys. J., 768, 185

Steiner, J. F., McClintock, J. E., \& Narayan, R., 2013, Astrophys. J., 762, 104

Svensson, R., \& Zdziarski, A. A., 1994, Astrophys. J., 436, 599

Tanaka, Y., \& Lewin, W. H. G. 1995, X-ray Binaries, 126

Vadawale, S. V., Rao, A. R., Nandi, A., \& Chakrabarti, S. K., 2001, Astron. Astrophys., 370, L17

Wu, K., et al. 2002, Astrophys. J., 565, 1161

Zurita, C., Sánchez-Fernández, C., Casares, J., et al., 2002, Mon. Not. R. Astron. Soc., 334, 999

This 2-column preprint was prepared with the AAS $\mathrm{LAT}_{\mathrm{E}} \mathrm{X}$ macros v5.2. 